\begin{tabular}{cc|c}
\hline Tar. Bil. Der. & Journal of Agricultural Sciences \\
& $\begin{array}{c}\text { Dergi web sayfası: } \\
\text { www.agri.ankara.edu.tr/dergi }\end{array}$ & Journal homepage: \\
& www.agri.ankara.edu.tr/journal
\end{tabular}

\title{
Buğday ve Arpada Farklı Toprak Nem Düzeylerinde Bazı Ekim Öncesi Tohum Uygulamalarının Etkinliği
}

\author{
Bekir ATAR ${ }^{\mathrm{a}}$, Burhan KARA \\ a Süleyman Demirel Üniversitesi, Tarımsal Araştırma ve Uygulama Merkezi, Isparta, TÜRKIYE \\ ${ }^{b}$ Süleyman Demirel Üniversitesi, Ziraat Fakültesi, Tarla Bitkileri Bölümü, Isparta, TÜRKIYE
}

\section{ESER BILGISİ}

Araștırma Makalesi

DOI: 10.1501/Tarimbil_0000001310

Sorumlu Yazar: Bekir ATAR, E-posta: bekiratar@sdu.edu.tr, Tel: +90 (246) 2114889

Geliş Tarihi: 08 Şubat 2014, Düzeltmelerin Gelişi: 04 Mayıs 2014, Kabul: 05 Haziran 2014

\section{ÖZET}

Araştırma beş farklı toprak nem düzeylerinde (tarla kapasitesi- $\% 100-\mathrm{S}_{1}$, tarla kapasitesinin \% 88'i-S $\mathrm{S}_{2}, \% 76$ 's1-S $\%$ 64'ü- $\mathrm{S}_{4}$ ve $\% 52$ 'si-solma noktası- $\mathrm{S}_{5}$ ) ekim öncesi tohum uygulamalarının (hidropriming, 30 ve 60 ppm GA ) çimlenme ve fide gelişimine etkilerinin belirlenmesi amacıyla yürütülmüştür. Çalışmada Soyer-2002 ekmeklik buğday ve Ç1ldır-2002 arpa çeşitleri kullanılmıştır. $\mathrm{GA}_{3}$ uygulamalarının etkisi genel olarak her iki cinste de ilk çimlenme süresini, yüzde elli çıkış süresini ve ortalama çıkış süresini azaltmış, çimlenme indeksini ve fide sap boyunu ise arttırmıştır. 30 ppm $\mathrm{GA}_{3}$ uygulamasında elde edilen ortalama değerler 60 ppm GA 3 uygulamasına göre daha yüksek olmuştur. İncelenen parametreler bakımından buğdayda en iyi sonuçlar $\mathrm{S}_{2}$ ve $\mathrm{S}_{3}$ toprak nem düzeylerinde belirlenirken, arpada $\mathrm{S}_{1}$ nem düzeyinde bulunmuştur. Anahtar Kelimeler: Buğday; Arpa; Çimlenme; Tohum uygulaması; Toprak nem düzeyleri

\section{Efficiency of Some Seed Priming in Different Soil Moisture Contents in Wheat and Barley}

\section{ARTICLE INFO}

Research Article

Corresponding Author: Bekir ATAR, E-mail: bekiratar@sdu.edu.tr, Tel: +90 (246) 2114889

Received: 08 February 2014, Received in Revised Form: 04 May 2014, Accepted: 05 June 2014

\begin{abstract}
The research was conducted to determine the effects of different soil moisture contents $\left(\mathrm{I}_{100}\right.$ full irrigation- $\mathrm{S}_{1}, \mathrm{I}_{88}: 88 \%$ of full irrigation- $\mathrm{S}_{2}, \mathrm{I}_{76}: 76 \%$ of full irrigation- $\mathrm{S}_{3}, \mathrm{I}_{64}: 64 \%$ of full irrigation- $\mathrm{S}_{4}$ and $\mathrm{I}_{52}: 52 \%$ of full irrigation-wilting point- $\mathrm{S}_{5}$ ) and seed priming ((hidropriming, 30 and $60 \mathrm{ppm} \mathrm{GA}$ ) on germination and seedling growing. The species of Soyer-2002 bread wheat and Çıldır-2002 barley was used as cultivars. Generally, effect on examined characteristics of $\mathrm{GA}_{3}$ were positived, and it was decreased to beginning germination periods, germination periods $50 \%$ and average germination periods, and increased to seedling height and germination index in both species. Research results in $30 \mathrm{ppm}$ $\mathrm{GA}_{3}$ treatment were higher than $60 \mathrm{ppm} \mathrm{GA}$. While the highest results in wheat was determined in $\mathrm{S}_{2}$ and $\mathrm{S}_{3}$ moisture contents, the highest results in barley was obtained from $\mathrm{S}_{1}$ moisture content.
\end{abstract}

Keywords: Wheat; Barley; Germination; Priming; Soil moisture levels 


\section{Giriş}

Doğrudan tarlaya ekilen tohumların eksiksiz, eşzamanlı ve güçlü bir şekilde toprak yüzüne çıkarak sağlıklı fideler oluşturması yüksek verim düzeyine ulaşmada önemli faktörlerden birisidir (Wurr \& Fellows 1983). Çıkış döneminde karşılaşılan çevresel faktörlerin başında toprak sıcaklığı, toprak nemi, oksijen seviyesi ile hastalık ve zararlılar gelmektedir. Düşük toprak nem düzeyinin çıkışı geciktirdiği bilinmektedir. Özellikle geniş ekim alanlarına sahip olan tarla bitkilerinde çevresel faktörlerin değiştirilmesi oldukça güçtür. 'Ekim öncesi tohum uygulamaları' diğer bir ifadeyle 'Seed Priming' bu dönemde oluşacak zararın azaltılmasında faydalı bir yöntem olarak kullanılmaktadır.

Tohum uygulamaları, tohumların ozmotik bir çözelti ya da su içerisinde çimlenmenin ilk aşaması tamamlanıncaya kadar su alımına izin veren, ancak kökçüğün çıkışına izin vermeyen uygulama olarak tanımlanmaktadır (Heydecker \& Coolbear 1977; Khan 1992). Yöntem uygun bitki sıklığını sağlamak için, tohumların hızlı ve üniform çıkış göstermesinde etkili olmaktadır (Harris et al 2005). Buğday'da priming uygulamalarının fide çıkış hızını (Farooq et al 2007), fide sap ve kök uzunluğunu, fide kök ve sap kuru ağırlığını (Basra et al 2005), erken çiçeklenme ve hasadı ile azot kullanım etkinliğini (Harris et al 2001), geç ekimlerde tane ve saman verimi ile hasat indeksini (Farooq et al 2007) ve verimi (Shahzad et al 2007) arttırdığı belirtilmiştir. Çıkış dönemindeki toprak nemi eksikliği olumsuz çevre koşullarının başında gelmektedir. Bu olumsuzluğunun etkisini azaltmaya yönelik olarak bitki büyüme düzenleyici içerikli tohum uygulamalarının tohumun çimlenme ve çıkış özelliklerine olumlu etki ettiği belirtilmektedir (Afzal et al 2005; Atar 2010). Tahıl yetiştiriciliğinde yüksek verime ulaşmada iklim ve toprak özellikleri belirleyici rol oynamaktadır. $\mathrm{Bu}$ çalışma; farklı toprak nem düzeylerinde ekim öncesi tohum uygulamalarının buğday ve arpanın çimlenme ve fide gelişimine etkisini araştırmak amaciyla yürütülmüştür.

\section{Materyal ve Yöntem}

\subsection{Tohum uygulamalart}

Araştırma Süleyman Demirel Üniversitesi Ziraat Fakültesi arazisinde, Soyer-2002 ekmeklik buğday ve Çıldır-2002 arpa çeşitleri kullanılarak 2012 yılında yürütülmüştür. Tohumlar uygulamalardan önce $\% 5$ Sodyum Hipoklorit $(\mathrm{NaOCl}$ ) ile dezenfekte edilerek, saf su ile yıkanmıştır. Temizlenen tohumlar kontrol (uygulaması), saf su (hidropriming), $30 \mathrm{ve}$ 60 ppm GA 3 içerikli 0.5 litrelik çözeltilerde 5 saat süreyle 1slatılmıştır (Khan 1992; Afzal et al 2005). Uygulama sonunda tohumlar saf su ile yıkanıp yüzey kurutması yapılarak oda sıcaklığında ve gölgede yaklaşık önceki nem içeriklerine ulaşıncaya kadar kurutulmuştur.

\subsection{Toprak nem düzeyi ve ekim}

Ekim öncesi siltli, killi ve tınlı yapıdaki tarla toprağı elenerek temiz ve homojen hale getirilmiştir. Ekim için $15 \times 15 \times 40 \mathrm{~cm}$ ebatlarında saksılar kullanılmış, her bir saksıya $6 \mathrm{~kg}$ toprak konulacak şekilde düzenlenmiştir. Alınan örneklerde toprak nem içeriği, tarla kapasitesi ve solma noktaları laboratuvar ortamında tespit edilmiştir. Tarla Kapasitesi $\left(\mathrm{S}_{1}\right)$, tarla kapasitesinin \% 88'i $\left(\mathrm{S}_{2}\right)$, \% 76's1 $\left(\mathrm{S}_{3}\right), \%$ 64'ü $\left(\mathrm{S}_{4}\right)$ ve \% 52'si $\left(\mathrm{S}_{5}\right)$ - solma noktası (SN) olmak üzere beş farklı nem düzeyi belirlenmiştir. Belirlenen tarla kapasitesine göre her bir saksıya ilave edilecek su miktarları hesaplanarak toprağa iyice karıştırılmış, istenen nem düzeyine getirilmiştir. Her saksı kendi içerisinde 4 parsele bölünerek, kontrol ve uygulama yapılmış 10'ar adet tohum $3 \mathrm{~cm}$ derinliğe ekilmiştir. Ekimden sonra toprak nem düzeyinin korunması için saksılar hava giriş çıkışına izin verecek şekilde streç film ile kaplanmıştır. Deneme boyunca saksılardaki nem kaybı \% 2-4 düzeyinde olmuştur. Deneme bölgede buğday ekim ve çıkışlarının olduğu kasım ayı içerisinde yürütülmüştür. Saksılar üstü kapalı, güneş görmeyen ve dış çevreye açık ortamda tutulmuştur. Denemenin yürütüldüğü dönemde ortalama hava sicaklığ $18.7^{\circ} \mathrm{C}, 5 \mathrm{~cm}$ toprak sicaklığ $19.8^{\circ} \mathrm{C}$ ve 10 cm toprak sicaklığ $10.2^{\circ} \mathrm{C}$ olmuştur. 


\section{3. Çimlenme testleri}

Ekimden itibaren çıkışlar günlük olarak sayılmış, aşağıdaki eşitlikler kullanılarak çimlenme ile ilgili parametreler belirlenmiştir.

$$
G P=\left(\sum n_{j} / N\right) 100
$$

Eşitlikte; GP, çimlenme yüzdesi; $n_{\mathrm{j},}$ çimlenen tohum sayısı ve $\mathrm{N}$ ise ekilen tohum sayısıdır. İlk çıkış süresi (FET), ekimden itibaren çıkışın görüldüğü gün ilk çimlenme süresi olarak belirlenmiştir. Yüzde elli çıkış süresi $\left(\mathrm{T}_{50}\right)$, aşağıda belirtilen eşitlik kullanılarak belirlenmiştir (Coolbear et al 1984 \& Farooq 2005).

$$
T 50=t_{i}+\left[\left((N / 2)-n_{j}\right)\left(t_{j}-t_{i}\right)\right] / n_{j}-n_{i}
$$

Eşitlikte; $\mathrm{N}$ toplam çimlenen tohum sayıs1, $n_{i}$ ve $n_{j}$ birbirini takip eden günlerdeki $\left(t_{i}\right.$ ve $\left.t_{j}\right)$ çimlenen tohum sayısını göstermektedir. Burada $\mathrm{n}_{\mathrm{i}}<\mathrm{N} / 2<\mathrm{n}_{\mathrm{j}}$ 'dir. Ortalama çıkış süresi (MGT), Ellis $\&$ Roberts (1981) tarafından belirtilen eşitlik esas alınarak belirlenmiştir.

$$
M G T=\left(\sum t_{i}-n_{i}\right) / \sum n_{i}
$$

Eşitlikte; MGT, ortalama çıkış üresini; $n_{i}$ ise ekimden itibaren $t_{i}$ gününde çıkan bitki sayısını göstermektedir. Çimlenme indeksi (GI), Resmi Tohum Analistleri Derneği (AOSA 1983) fide değerlendirme esasına göre hesaplanmıştır.

$$
G I=\left[\left(n_{i} / t_{i}\right)+\ldots . .+\left(n_{n} / t_{n}\right)\right]
$$

Eşitlikte; GI, çimlenme indeksini; $n_{i}$, ekimde itibaren $t_{i}$ gününde çimlenen tohum sayısını göstermektedir. Denemede tohum çıkış sayımları yirmibirinci günde sona erdirilmiştir. Aynı gün deneme saksıları su dolu kapların içine gömülerek 6-7 saat bekletilmiş toprakların yumuşaması sağlanmış ve söküm yapılmıştır. Temizlenen fideler köklerin tohumla birleştiği noktadan ayrılarak fide sap boyu (FSB), fide kök boyu (FKB) cm olarak belirlenmiştir. Fide sap ve kök kısımları birleştirilip fide yaş ağırlı̆̆ (FYA) belirlendikten sonra $70^{\circ} \mathrm{C}$ firında sabit ağırlığa gelinceye kadar bekletilerek fide kuru ağırlığı (FKA) g olarak belirlenmiştir. Çalışmada elde edilen veriler, JUMP istatistik paket programından faydalanılarak;
Tesadüf parsellerinde faktöriyel düzende varyans analizleri yapılmış, ortalamalar arasındaki farklılıklar DUNCAN testine göre karşılaştırılmıştır.

\section{Bulgular ve Tartışma}

Toprak nem düzeylerinde tohum uygulamalarının çimlenme ve fide gelişimi üzerine etkileri Çizelge 1 ve 2'de verilmiştir. Buğday çimlenme oranında en yüksek değerler $\mathrm{S}_{3}$ nem düzeyinde, fide kuru ağırlığ ise $S_{1}$ nem düzeyinin 30 ppm $\mathrm{GA}_{3}$ uygulamasında belirlenmiştir. Araştırılan diğer özelliklerdeki olumlu etkiler $S_{2}$ ve $S_{3}$ nem düzeyi ile $60 \mathrm{ppm}$ $\mathrm{GA}_{3}$ uygulamasında yoğunlaşmıştır. Arpada ise çimlenme yüzdesi buğdaya göre daha yüksek bulunurken en düşük değer $S_{1}$ nem düzeyinde ve 60 ppm $\mathrm{GA}_{3}$ uygulamasında belirlenmiştir. Çıkış hızı ile ilgili özellikler ve çimlenme indeksi bakımından $S_{1}$ ve $S_{2}$ nem düzeyinin tohum uygulamalarında daha olumlu etkiler gözlenirken, bu durum $\mathrm{S}_{2}$ nem düzeyi ve 60 ppm $\mathrm{GA}_{3}$ uygulamasında belirgindir. Arpada en yüksek fide sap boyu $\mathrm{S}_{3}$ toprak nem

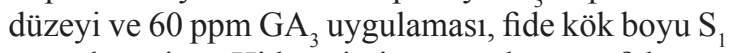
nem düzeyi ve Hidropriming uygulaması, fide yaş ağırlığı $\mathrm{S}_{1}$ nem düzeyi ve 60 ppm GA $\mathrm{G}_{3}$ uygulamas1, fide kuru ağırlığı ise $S_{2}$ nem düzeyi ve 60 ppm $\mathrm{GA}_{3}$ uygulamalarında belirlenmiştir.

Buğdayda çimlenme yüzdesi $\mathrm{S}_{3}$ nem düzeyinde \% 5 daha yüksek çıkarken, en hızlı çıkış süreleri ve büyüme özellikleri $\mathrm{S}_{2}$ nem düzeyinde belirlenmiştir. Bunu $S_{3}$ nem düzeyi takip etmiştir. $S_{1}$ nem düzeyinde fide çıkış hızı ile ilgili veriler daha olumlu iken, $\mathrm{S}_{4}$ nem düzeyinde fide gelişimi ile ilgili özellikler daha yüksek bulunmuştur. Genel olarak incelenen dokuz özelliğin altısında en düşük değerleri $\mathrm{S}_{5}$ nem düzeyinde tespit edilmiştir (Çizelge 1). Arpada ise incelenen özelliklere toprak nem düzeylerinin etkisi belirgin olmuştur. En hızlı çıkış süreleri ve büyüme özellikleri tarla kapasitesinde $\left(\mathrm{S}_{1}\right)$ belirlenmiş, toprak nem düzeyindeki azalmaya bağlı olarak değerlerde anlamlı bir azalma görülmüştür (Çizelge 2).

Buğday ve arpada tohum uygulamaları bakımından $\mathrm{GA}_{3}$ uygulamalarının incelenen özelliklere olumlu etkisi belirgindir (Çizelge 1 ve 2). Tohum uygulamalarında en hızlı çıkış değerleri ve büyüme özellikleri genel olarak 60 ppm $\mathrm{GA}_{3}$ uygulamalarında elde edilmiş olsa da, ortalamalara 
Çizelge 1- Tohum uygulamaları ve toprak nem düzeylerinin buğdayın çimlenme özellikleri üzerine etkileri Table 1- Effects of seed priming and soil moisture levels on germination of wheat

\begin{tabular}{|c|c|c|c|c|c|c|c|c|c|c|}
\hline \multicolumn{2}{|c|}{ Nem düzeyi/ Primings } & \multirow{2}{*}{$\frac{G P}{81.3 \mathrm{bc}^{* *}}$} & \multirow{2}{*}{$\frac{F E T}{8.5 \mathrm{fg} * *}$} & \multirow{2}{*}{$\frac{T_{50}}{11.3 \mathrm{c}^{* *}}$} & \multirow{2}{*}{$\frac{M E T}{11.8 \mathrm{c}^{* *}}$} & \multirow{2}{*}{$\frac{G I}{0.57 h_{1 j} * *}$} & \multirow{2}{*}{$\frac{F S B}{8.0 \mathrm{ij}^{* *}}$} & \multirow{2}{*}{$\begin{array}{l}F K B \\
10.3 \mathrm{gh}^{* *}\end{array}$} & \multirow{2}{*}{$\begin{array}{l}F Y A \\
0.156 \mathrm{~d}-\mathrm{g}^{* *}\end{array}$} & \multirow{2}{*}{$\begin{array}{l}F K A \\
0.021 \mathrm{f}^{* *}\end{array}$} \\
\hline & Kontrol & & & & & & & & & \\
\hline \multirow{3}{*}{ S1 } & Hidropriming & $87.5 \mathrm{bc}$ & $7.5 \mathrm{~h} 1$ & $10.0 \mathrm{de}$ & $10.8 \mathrm{~d}$ & $0.68 \mathrm{f}$ & $7.8 \mathrm{ij}$ & $10.9 \mathrm{fg}$ & 0.172 de & $0.022 \mathrm{ef}$ \\
\hline & $30 \mathrm{ppm} \mathrm{GA}_{3}$ & $93.8 \mathrm{ab}$ & $6.5 \mathrm{j}$ & $7.4 \mathrm{~h} 1$ & $8.5 \mathrm{fg}$ & $0.93 \mathrm{abc}$ & $10.6 \mathrm{c}$ & $12.0 \mathrm{e}$ & $0.216 \mathrm{ab}$ & $0.029 \mathrm{a}$ \\
\hline & $60 \mathrm{ppm} \mathrm{GA}$ & $75.0 \mathrm{c}$ & $7.0 \mathrm{ij}$ & $10.7 \mathrm{~cd}$ & $10.7 \mathrm{~d}$ & $0.59 \mathrm{gh} 1$ & 9.6 ef & 10.9 fgh & $0.172 \mathrm{~d}$ & $0.023 \mathrm{def}$ \\
\hline \multirow{4}{*}{$\mathrm{S} 2$} & Kontrol & $93.8 \mathrm{ab}$ & $8.0 \mathrm{gh}$ & $8.7 \mathrm{fg}$ & $9.3 \mathrm{e}$ & $0.83 \mathrm{de}$ & $9.8 \mathrm{de}$ & $13.5 \mathrm{ab}$ & $0.240 \mathrm{a}$ & $0.027 \mathrm{ab}$ \\
\hline & Hidropriming & $93.8 \mathrm{ab}$ & $8.0 \mathrm{gh}$ & $8.5 \mathrm{fg}$ & $9.0 \mathrm{ef}$ & 0.85 cde & $9.1 \mathrm{efg}$ & $13.4 \mathrm{abc}$ & $0.169 \mathrm{de}$ & 0.024 cde \\
\hline & $30 \mathrm{ppm} \mathrm{GA}$ & $100.0 \mathrm{a}$ & $7.0 \mathrm{ij}$ & $7.9 \mathrm{gh}$ & $8.4 \mathrm{fg}$ & $0.96 \mathrm{ab}$ & $10.7 \mathrm{bc}$ & $13.0 \mathrm{bcd}$ & $0.201 \mathrm{bc}$ & $0.026 \mathrm{bc}$ \\
\hline & 60 ppm GA 3 & $87.5 \mathrm{abc}$ & $5.5 \mathrm{k}$ & 6.91 & $8.3 \mathrm{~g}$ & 0.86 cde & $12.2 \mathrm{a}$ & $14.4 \mathrm{a}$ & $0.235 \mathrm{a}$ & $0.027 \mathrm{ab}$ \\
\hline \multirow{4}{*}{ S3 } & Kontrol & $100.0 \mathrm{a}$ & $9.5 \mathrm{de}$ & $9.9 \mathrm{de}$ & $10.3 \mathrm{~d}$ & $0.78 \mathrm{de}$ & $9.0 \mathrm{fgh}$ & $13.1 \mathrm{bcd}$ & $0.205 \mathrm{~b}$ & $0.023 \mathrm{def}$ \\
\hline & Hidropriming & $93.8 \mathrm{ab}$ & $8.0 \mathrm{gh}$ & 9.4 ef & $9.6 \mathrm{e}$ & $0.80 \mathrm{de}$ & $8.3 \mathrm{~h} 1$ & $12.2 \mathrm{de}$ & $0.180 \mathrm{~cd}$ & $0.022 \mathrm{ef}$ \\
\hline & $30 \mathrm{ppm} \mathrm{GA}$ & $100.0 \mathrm{a}$ & $8.0 \mathrm{gh}$ & $8.7 \mathrm{fg}$ & $9.3 \mathrm{e}$ & $0.88 \mathrm{bcd}$ & $10.9 \mathrm{bc}$ & $13.1 \mathrm{bcd}$ & $0.221 \mathrm{ab}$ & $0.025 \mathrm{bcd}$ \\
\hline & $60 \mathrm{ppm} \mathrm{\textrm {GA } _ { 3 }}$ & $100.0 \mathrm{a}$ & $7.0 \mathrm{ij}$ & $7.5 \mathrm{~h} 1$ & $8.2 \mathrm{~g}$ & $1.00 \mathrm{a}$ & $12.4 \mathrm{a}$ & $12.5 \mathrm{cde}$ & $0.203 b c$ & $0.024 \mathrm{bcd}$ \\
\hline \multirow{4}{*}{ S4 } & Kontrol & $87.5 \mathrm{abc}$ & $10.0 \mathrm{~d}$ & $10.4 \mathrm{~d}$ & $10.9 \mathrm{~d}$ & $0.65 \mathrm{fgh}$ & $8.7 \mathrm{gh}$ & $11.6 \mathrm{ef}$ & 0.147 efg & $0.017 \mathrm{~g}$ \\
\hline & Hidropriming & $87.5 \mathrm{abc}$ & $10.0 \mathrm{~d}$ & $10.6 \mathrm{~cd}$ & $10.8 \mathrm{~d}$ & $0.65 \mathrm{fgh}$ & $8.3 \mathrm{~h} 1$ & 10.2 ghı & $0.133 \mathrm{gh}$ & $0.018 \mathrm{~g}$ \\
\hline & $30 \mathrm{ppm} \mathrm{G}_{3}$ & $100.0 \mathrm{a}$ & $9.0 \mathrm{ef}$ & $10.2 \mathrm{de}$ & $10.4 \mathrm{~d}$ & $0.78 \mathrm{e}$ & $10.4 \mathrm{~cd}$ & $9.9 \mathrm{~h} 1$ & $0.158 \mathrm{def}$ & $0.021 \mathrm{f}$ \\
\hline & $60 \mathrm{ppm} \mathrm{G}_{3}$ & $87.5 \mathrm{abc}$ & 9.0 ef & 10.2 de & $10.6 \mathrm{~d}$ & $0.67 \mathrm{fg}$ & $11.3 \mathrm{~b}$ & 9.21 & $0.141 \mathrm{fg}$ & $0.021 \mathrm{f}$ \\
\hline \multirow{4}{*}{ S5 } & Kontrol & $93.8 \mathrm{ab}$ & $13.0 \mathrm{~b}$ & $14.0 \mathrm{~b}$ & $14.3 \mathrm{~b}$ & $0.531 \mathrm{jk}$ & 4.51 & $6.5 \mathrm{j}$ & $0.079 \mathrm{j}$ & 0.0111 \\
\hline & Hidropriming & $81.2 \mathrm{bc}$ & $14.0 \mathrm{a}$ & $15.5 \mathrm{a}$ & $15.5 \mathrm{a}$ & 0.371 & 4.61 & $6.8 \mathrm{j}$ & $0.083 \mathrm{j}$ & $0.013 \mathrm{~h} 1$ \\
\hline & $30 \mathrm{ppm} \mathrm{GA}$ & $81.2 \mathrm{bc}$ & $11.0 \mathrm{c}$ & $14.1 \mathrm{~b}$ & $14.4 \mathrm{~b}$ & $0.45 \mathrm{kl}$ & $5.8 \mathrm{k}$ & $6.7 \mathrm{j}$ & $0.109 \mathrm{~h} 1$ & $0.015 \mathrm{~h} 1$ \\
\hline & $60 \mathrm{ppm} \mathrm{GA}$ & $87.5 \mathrm{abc}$ & $11.0 \mathrm{c}$ & $13.5 \mathrm{~b}$ & $13.8 \mathrm{~b}$ & $0.49 \mathrm{jk}$ & $7.3 \mathrm{j}$ & $6.9 \mathrm{j}$ & $0.091 \mathrm{ij}$ & $0.016 \mathrm{gh}$ \\
\hline \multicolumn{11}{|c|}{ Nem düzeyleri } \\
\hline & S1 & $84.4 \mathrm{c} * *$ & $7.4 d^{* *}$ & $9.9 \mathrm{c} *$ & $10.5 \mathrm{~b}^{* *}$ & $0.69 \mathrm{~b}^{* *}$ & $9.0 \mathrm{c}^{* *}$ & $11.0 \mathrm{c}^{* *}$ & $0.179 b^{* *}$ & $0.024 \mathrm{~b}^{* *}$ \\
\hline & $\mathrm{S} 2$ & $93.8 \mathrm{ab}$ & $7.1 \mathrm{~d}$ & $8.0 \mathrm{e}$ & $8.8 \mathrm{~d}$ & $0.88 \mathrm{a}$ & $10.4 \mathrm{a}$ & $13.6 \mathrm{a}$ & $0.211 \mathrm{a}$ & $0.026 \mathrm{a}$ \\
\hline & $\mathrm{S} 3$ & $98.5 \mathrm{a}$ & $8.1 \mathrm{c}$ & $8.9 \mathrm{~d}$ & $9.4 \mathrm{c}$ & $0.87 \mathrm{a}$ & $10.2 \mathrm{a}$ & $12.7 \mathrm{~b}$ & $0.202 \mathrm{a}$ & $0.023 \mathrm{~b}$ \\
\hline & $\mathrm{S} 4$ & $90.6 \mathrm{bc}$ & $9.5 \mathrm{~b}$ & $10.4 \mathrm{~b}$ & $10.7 \mathrm{~b}$ & $0.69 \mathrm{~b}$ & $9.7 \mathrm{~b}$ & $10.2 \mathrm{~d}$ & $0.144 \mathrm{c}$ & $0.019 \mathrm{c}$ \\
\hline & S5 & $85.9 \mathrm{c}$ & $12.3 \mathrm{a}$ & $14.3 \mathrm{a}$ & $14.5 \mathrm{a}$ & $0.46 \mathrm{c}$ & $5.6 \mathrm{~d}$ & $6.7 \mathrm{e}$ & $0.091 \mathrm{~d}$ & $0.014 \mathrm{~d}$ \\
\hline \multicolumn{11}{|c|}{ Primings } \\
\hline \multicolumn{2}{|c|}{ Kontrol } & $91.3 \mathrm{ab}^{*}$ & $9.8 \mathrm{a}^{*}$ & $10.9 a^{*}$ & $11.3 \mathrm{a}^{*}$ & $0.67 \mathrm{c}^{* *}$ & $8.0 \mathrm{c}^{* *}$ & 11.0 & $0.165 \mathrm{~b}$ & $0.020 \mathrm{~b}^{*}$ \\
\hline \multicolumn{2}{|c|}{ Hidropriming } & $88.8 \mathrm{~b}$ & $9.5 \mathrm{a}$ & $10.8 \mathrm{a}$ & $11.1 \mathrm{a}$ & $0.67 \mathrm{c}$ & $7.6 \mathrm{~d}$ & 10.7 & $0.147 \mathrm{c}$ & $0.020 \mathrm{~b}$ \\
\hline \multicolumn{2}{|c|}{$30 \mathrm{ppm} \mathrm{GA}$} & $95.0 \mathrm{a}$ & $8.3 \mathrm{~b}$ & $9.7 \mathrm{~b}$ & $10.2 \mathrm{~b}$ & $0.80 \mathrm{a}$ & $9.7 \mathrm{~b}$ & 10.9 & $0.181 \mathrm{a}$ & $0.023 \mathrm{a}$ \\
\hline \multicolumn{2}{|c|}{$60 \mathrm{ppm} \mathrm{GA}_{3}$} & $87.5 \mathrm{~b}$ & $7.9 \mathrm{~b}$ & $9.8 \mathrm{~b}$ & $10.3 \mathrm{~b}$ & $0.72 \mathrm{~b}$ & $10.6 \mathrm{a}$ & 10.8 & $0.168 \mathrm{~b}$ & $0.022 \mathrm{a}$ \\
\hline \multicolumn{2}{|c|}{ CV (\%) } & 8.7 & 6.7 & 5.2 & 4.0 & 7.7 & 4.6 & 5.4 & 9.4 & 7.1 \\
\hline
\end{tabular}

**, $\mathrm{P} \leq 0.01 ; *, \mathrm{P} \leq 0.05$ düzeyinde önemli; $\mathrm{S}_{1}$, tarla kapasitesi; $\mathrm{S}_{2}$, tarla kapasitesinin \% 88'i; $\mathrm{S}_{3}$, tarla kapasitesinin \% 76's1; $\mathrm{S}_{4}$, tarla kapasitesinin \% 64'ü; $S_{5}$, tarla kapasitesinin $\% 52$ 'i; GP, çimlenme yüzdesi; FET, ilk çimlenme süresi; $T_{50}, \% 50$ çıkış süresi; MET, ortalama çıkış süresi; GI, çimlenme indeksi; FSB, fide sap boyu; FKB, fide kök boyu; FYA, fide yaş ağırlığı; FKA, fide kuru ağırlığı 
Çizelge 2- Tohum uygulamaları ve toprak nem düzeylerinin arpanın çimlenme özellikleri üzerine etkileri Table 2- Effects of seed priming and soil moisture levels on germination of barley

\begin{tabular}{|c|c|c|c|c|c|c|c|c|c|c|}
\hline \multicolumn{2}{|c|}{ Nem düzeyi/Primings } & \multirow{2}{*}{$\frac{G P}{100.0 \mathrm{a}^{*}}$} & \multirow{2}{*}{$\frac{F E T}{8.0 \mathrm{ef}^{* *}}$} & \multirow{2}{*}{$\frac{T_{50}}{8.11^{* *}}$} & \multirow{2}{*}{$\frac{M E T}{8.7 \mathrm{jkl}{ }^{* *}}$} & \multirow{2}{*}{$\frac{G I}{0.94 \mathrm{~b}^{* *}}$} & \multirow{2}{*}{$\frac{F S B}{11.7 \mathrm{efg}^{* *}}$} & \multirow{2}{*}{$\frac{F K B}{15.0 \mathrm{bc}^{* *}}$} & \multirow{2}{*}{$\begin{array}{l}F Y A \\
0.330 \mathrm{ab}^{* *}\end{array}$} & \multirow{2}{*}{$\frac{F K A}{0.033 \mathrm{a}-\mathrm{f}^{* *}}$} \\
\hline & Kontrol & & & & & & & & & \\
\hline \multirow{3}{*}{ S1 } & Hidropriming & $100.0 \mathrm{a}$ & $7.0 \mathrm{~g}$ & 7.81 & $8.4 \mathrm{kl}$ & $0.97 \mathrm{ab}$ & $12.6 \mathrm{c}-\mathrm{g}$ & $16.6 \mathrm{a}$ & $0.278 \mathrm{cde}$ & $0.036 \mathrm{ab}$ \\
\hline & 30 ppm $\mathrm{GA}_{3}$ & $93.8 \mathrm{~b}$ & $7.0 \mathrm{~g}$ & 7.81 & $8.3 \mathrm{kl}$ & $0.93 \mathrm{~b}$ & $13.0 \mathrm{bcd}$ & $15.7 \mathrm{ab}$ & $0.340 \mathrm{ab}$ & $0.035 \mathrm{abc}$ \\
\hline & $60 \mathrm{ppm} \mathrm{GA}{ }_{3}$ & $87.5 \mathrm{c}$ & $7.0 \mathrm{~g}$ & 8.01 & $8.91 \mathrm{jk}$ & $0.81 \mathrm{~cd}$ & $13.7 \mathrm{bc}$ & $15.6 \mathrm{ab}$ & $0.357 \mathrm{a}$ & $0.035 \mathrm{ab}$ \\
\hline \multirow{4}{*}{$\mathrm{S} 2$} & Kontrol & $93.8 \mathrm{~b}$ & $8.0 \mathrm{ef}$ & $9.6 \mathrm{ef}$ & $10.1 \mathrm{e}-\mathrm{h}$ & $0.78 \mathrm{fg}$ & $12.9 \mathrm{~b}-\mathrm{e}$ & $13.3 \mathrm{de}$ & $0.330 \mathrm{ab}$ & 0.034 a-e \\
\hline & Hidropriming & $100.0 \mathrm{a}$ & 8.0 ef & $8.8 \mathrm{gh}$ & $9.6 \mathrm{~h} 1$ & $0.86 \mathrm{c}$ & $12.4 \mathrm{~d}-\mathrm{g}$ & $15.0 \mathrm{bc}$ & $0.328 \mathrm{ab}$ & $0.034 \mathrm{a}-\mathrm{e}$ \\
\hline & 30 ppm GA & $100.0 \mathrm{a}$ & $7.0 \mathrm{~g}$ & $8.2 \mathrm{~h} 1$ & $8.6 \mathrm{kl}$ & $0.95 \mathrm{~b}$ & $12.5 \mathrm{c}-\mathrm{g}$ & $14.1 \mathrm{~cd}$ & $0.319 \mathrm{abc}$ & $0.034 \mathrm{a}-\mathrm{e}$ \\
\hline & $60 \mathrm{ppm} \mathrm{GA}$ & $100.0 \mathrm{a}$ & $7.0 \mathrm{~g}$ & 7.71 & 7.91 & $1.03 \mathrm{a}$ & $12.6 \mathrm{~b}-\mathrm{g}$ & $15.2 \mathrm{bc}$ & $0.337 \mathrm{ab}$ & $0.037 \mathrm{a}$ \\
\hline \multirow{4}{*}{$\mathrm{S} 3$} & Kontrol & $100.0 \mathrm{a}$ & $9.0 \mathrm{~cd}$ & $10.5 \mathrm{c}$ & $10.7 \mathrm{def}$ & $0.76 \mathrm{ef}$ & $10.31 \mathrm{j}$ & $12.6 \mathrm{ef}$ & 0.274 cde & $0.031 \mathrm{ef}$ \\
\hline & Hidropriming & $100.0 \mathrm{a}$ & $8.5 \mathrm{de}$ & $9.7 \mathrm{def}$ & $10.0 \mathrm{fgh}$ & $0.81 \mathrm{cde}$ & $11.5 \mathrm{gh} 1$ & $14.3 \mathrm{~cd}$ & $0.301 \mathrm{~b}-\mathrm{e}$ & $0.032 \mathrm{~b}-\mathrm{f}$ \\
\hline & $30 \mathrm{ppm} \mathrm{GA}_{3}$ & $100.0 \mathrm{a}$ & $8.5 \mathrm{de}$ & $9.2 \mathrm{fg}$ & $9.7 \mathrm{gh}$ & $0.84 \mathrm{c}$ & $13.3 \mathrm{bcd}$ & $12.9 \mathrm{ef}$ & $0.301 \mathrm{~b}-\mathrm{e}$ & $0.032 \mathrm{~b}-\mathrm{f}$ \\
\hline & $60 \mathrm{ppm} \mathrm{GA}$ & $100.0 \mathrm{a}$ & $9.0 \mathrm{~cd}$ & $9.3 \mathrm{fg}$ & $9.4 \mathrm{hij}$ & $0.85 \mathrm{c}$ & $15.4 \mathrm{a}$ & $13.4 \mathrm{de}$ & $0.312 \mathrm{a}-\mathrm{d}$ & $0.034 \mathrm{a}-\mathrm{f}$ \\
\hline \multirow{4}{*}{$\mathrm{S} 4$} & Kontrol & $100.0 \mathrm{a}$ & $10.0 \mathrm{~b}$ & $10.7 \mathrm{c}$ & $11.3 \mathrm{~cd}$ & $0.72 \mathrm{fgh}$ & $9.6 \mathrm{jk}$ & $14.1 \mathrm{~cd}$ & $0.297 \mathrm{~b}-\mathrm{e}$ & $0.031 \mathrm{def}$ \\
\hline & Hidropriming & $93.8 \mathrm{~b}$ & $10.0 \mathrm{~b}$ & $10.5 \mathrm{c}$ & $10.9 \mathrm{def}$ & 0.69 ghı & $10.4 \mathrm{hij}$ & $14.1 \mathrm{~cd}$ & $0.270 \mathrm{de}$ & $0.033 \mathrm{~b}-\mathrm{f}$ \\
\hline & 30 ppm $\mathrm{GA}_{3}$ & $100.0 \mathrm{a}$ & $9.5 \mathrm{bc}$ & $10.0 \mathrm{cde}$ & $10.4 \mathrm{efg}$ & 0.77 def & $12.7 \mathrm{~b}-\mathrm{f}$ & $12.7 \mathrm{ef}$ & $0.281 \mathrm{cde}$ & $0.031 \mathrm{c}-\mathrm{f}$ \\
\hline & $60 \mathrm{ppm} \mathrm{\textrm {GA } _ { 3 }}$ & $100.0 \mathrm{a}$ & $10.0 \mathrm{~b}$ & $10.3 \mathrm{cde}$ & $10.7 \mathrm{def}$ & $0.75 \mathrm{rf}$ & $13.8 \mathrm{~b}$ & $13.7 \mathrm{de}$ & $0.266 \mathrm{e}$ & $0.030 \mathrm{f}$ \\
\hline \multirow{4}{*}{ S5 } & Kontrol & $100.0 \mathrm{a}$ & $11.5 \mathrm{a}$ & $11.7 \mathrm{~b}$ & $11.9 \mathrm{bc}$ & 0.67 hij & 7.61 & $12.1 \mathrm{fg}$ & $0.207 \mathrm{f}$ & $0.024 \mathrm{~g}$ \\
\hline & Hidropriming & $100.0 \mathrm{a}$ & $12.0 \mathrm{a}$ & $12.2 \mathrm{ab}$ & $12.4 \mathrm{ab}$ & $0.64 \mathrm{ij}$ & $9.0 \mathrm{k}$ & $11.3 \mathrm{~g}$ & $0.187 \mathrm{f}$ & $0.023 \mathrm{~g}$ \\
\hline & $30 \mathrm{ppm} \mathrm{GA}$ & $100.0 \mathrm{a}$ & $12.0 \mathrm{a}$ & $12.0 \mathrm{~b}$ & $12.4 \mathrm{ab}$ & $0.65 \mathrm{ij}$ & $10.0 \mathrm{j}$ & $9.8 \mathrm{~h}$ & $0.201 \mathrm{f}$ & $0.025 \mathrm{~g}$ \\
\hline & $60 \mathrm{ppm} \mathrm{GA}$ & $100.0 \mathrm{a}$ & $12.0 \mathrm{a}$ & $12.6 \mathrm{ab}$ & $13.0 \mathrm{a}$ & $0.62 \mathrm{j}$ & $11.6 \mathrm{fgh}$ & $10.1 \mathrm{~h}$ & $0.191 \mathrm{f}$ & $0.025 \mathrm{~g}$ \\
\hline \multicolumn{11}{|c|}{ Nem düzeyleri } \\
\hline & $\mathrm{S} 1$ & $95.3 b^{*}$ & $7.3 \mathrm{e}^{* *}$ & $7.9 \mathrm{e}^{* *}$ & $8.6 \mathrm{e}^{* *}$ & $0.91 \mathrm{a}^{* *}$ & $12.8 \mathrm{a}^{* *}$ & $15.7 \mathrm{a}^{* *}$ & $0.326 \mathrm{a}^{* *}$ & $0.035 \mathrm{a}^{*}$ \\
\hline & S2 & $98.5 \mathrm{a}$ & $7.5 \mathrm{~d}$ & $8.6 \mathrm{~d}$ & $9.1 \mathrm{~d}$ & $0.91 \mathrm{a}$ & $12.6 \mathrm{~b}$ & $14.4 \mathrm{~b}$ & $0.329 \mathrm{a}$ & $0.035 \mathrm{a}$ \\
\hline & S3 & $100.0 \mathrm{a}$ & $8.8 \mathrm{c}$ & $9.7 \mathrm{c}$ & $10.0 \mathrm{c}$ & $0.82 \mathrm{~b}$ & $12.6 \mathrm{a}$ & $13.3 \mathrm{c}$ & $0.297 \mathrm{~b}$ & $0.032 \mathrm{~b}$ \\
\hline & S4 & $98.5 \mathrm{a}$ & $9.9 \mathrm{~b}$ & $10.4 \mathrm{~b}$ & $10.8 \mathrm{~b}$ & $0.73 \mathrm{c}$ & $11.6 \mathrm{~b}$ & $13.7 \mathrm{c}$ & $0.278 \mathrm{~b}$ & $0.031 \mathrm{~b}$ \\
\hline & S5 & $100.0 \mathrm{a}$ & $11.9 \mathrm{a}$ & $12.1 \mathrm{a}$ & $12.4 \mathrm{a}$ & $0.65 \mathrm{~d}$ & $9.6 \mathrm{c}$ & $10.8 \mathrm{~d}$ & $0.197 \mathrm{c}$ & $0.024 \mathrm{c}$ \\
\hline \multicolumn{11}{|c|}{ Primings } \\
\hline & Kontrol & 98.8 & $9.3 \mathrm{a}^{*}$ & $10.1 \mathrm{a}^{*}$ & $10.5 a^{*}$ & $0.77 c^{*}$ & $10.4 \mathrm{~d}^{* *}$ & $13.4 \mathrm{bc}^{*}$ & 0.289 & 0.030 \\
\hline & Hidropriming & 98.8 & $9.1 \mathrm{a}$ & $9.8 \mathrm{ab}$ & $10.3 \mathrm{ab}$ & $0.79 \mathrm{~b}$ & $11.2 \mathrm{c}$ & $14.3 \mathrm{a}$ & 0.273 & 0.031 \\
\hline & 30 ppm $\mathrm{GA}_{3}$ & 98.8 & $8.8 \mathrm{~b}$ & $9.4 \mathrm{c}$ & $9.9 \mathrm{c}$ & $0.83 \mathrm{a}$ & $12.3 \mathrm{~b}$ & $13.0 \mathrm{c}$ & 0.288 & 0.031 \\
\hline & $60 \mathrm{ppm} \mathrm{\textrm {GA } _ { 3 }}$ & 97.5 & $9.0 \mathrm{ab}$ & $9.6 \mathrm{bc}$ & $10.0 \mathrm{bc}$ & $0.81 \mathrm{ab}$ & $13.4 \mathrm{a}$ & $13.6 \mathrm{~b}$ & 0.293 & 0.032 \\
\hline \multicolumn{2}{|c|}{$\mathrm{CV}(\%)$} & 8.7 & 6.7 & 5.2 & 4.0 & 7.7 & 4.6 & 5.4 & 9.4 & 7.1 \\
\hline
\end{tabular}

**, $\mathrm{P} \leq 0.01 ; *, \mathrm{P} \leq 0.05$ düzeyinde önemli; $\mathrm{S}_{1}$, tarla kapasitesi; $\mathrm{S}_{2}$, tarla kapasitesinin \% 88’i; $\mathrm{S}_{3}$, tarla kapasitesinin \% 76’s1; $\mathrm{S}_{4}$, tarla kapasitesinin \% 64'ü; $S_{5}$, tarla kapasitesinin \% 52'si; GP, çimlenme yüzdesi; FET, ilk çimlenme süresi; $T_{50}, \% 50$ çıkış süresi; MET, ortalama çıkış süresi; GI, çimlenme indeksi; FSB, fide sap boyu; FKB, fide kök boyu; FYA, fide yaş ağırlığı; FKA, fide kuru ağırlığı 
bakıldığında 30 ppm $\mathrm{GA}_{3}$ uygulamalarının daha etkili olduğu görülmektedir. Buğday ve arpada kontrol ile hidropriming uygulamaları arasındaki farklılıklar genel olarak önemsiz bulunmuştur.

Buğdayda $\mathrm{S}_{1}$ nem düzeyinde $30 \mathrm{ppm} \mathrm{GA}_{3}$ uygulamas1 ve hidropriming uygulamalarında çimlenme oranının artması, İdris \& Aslam (1975) çalışmalarında belirttiği priming uygulamalarının stres şartlarında final çimlenme oranını arttırdığı sonucu ile benzerlik göstermektedir. $\mathrm{S}_{1}$ toprak nem düzeyinde 60 ppm $\mathrm{GA}_{3}$ uygulamasında çimlenme oranının düşük kalması, yüksek dozda $\mathrm{GA}_{3}$ uygulamasının yüksek toprak nemi ile birlikte tohumda çürümelere yol açtığ şeklinde yorumlanmıştır. $S_{5}$ toprak nem düzeyinde priming uygulamalarının etkili olmaması ve diğer nem düzeyindeki uygulamalarda bulunan yüksek değerler priming uygulamalarının etkinliğinin ortaya çıkmasında asgari nem varlığının önemli olduğunu ortaya koymaktadır. Fakat bu durum priming uygulamalarının uygun koşullarda etkili olmadığı (İdris \& Aslam 1975; Harris et al 2001; Giri \& Schillinger 2003; Basra et al 2005) görüşü ile uyuşmamaktadır.

Lindstrom et al (1976) tarla kapasitesinden itibaren azalan neme bağlı olarak çimlenme oranının azalma gösterdiğini bildirmişlerdir. Çalışmamızda ise en yüksek çimlenme oranları tarla kapasitesi ile solma noktası arasındaki toprak nem değerlerinde alınmış, tarla kapasitesinde nispeten daha düşük çimlenme oranları elde edilmiştir. Tarla kapasitesindeki topraklarda düşük oksijen seviyesinin (Özbingöl et al 1999) ve solma noktasında $\left(\mathrm{S}_{5}\right)$ ise düşük nem düzeyinin çimlenmeyi olumsuz etkilediği düşünülmektedir. Yeterli toprak nemi ve buna bağlı olarak yeterli oksijen seviyelerinde $\left(\mathrm{S}_{2}, \mathrm{~S}_{3}\right)$ çimlenme oranları yüksek bulunmuştur. $\mathrm{Bu}$ durum buğdayda net bir şekilde görülürken, arpada da benzer bir etki ortaya çıkmasına rağmen, $S_{5}$ nem düzeyinde final çimlenme oranı daha yüksek bulunması, arpanın nem düzeylerine daha toleranslı olduğu, kavuzlarının ortamdaki kısıtlı nemi alarak daha uzun süre muhafaza etmesinden kaynaklandığı düşünülmektedir.

Buğday ve arpada çıkış başlangıç süresinin, $\% 50$ çıkış zamanının ve ortalama çıkış süresinin tohum uygulamaları ile azaldığı, bu azalmanın $\mathrm{GA}_{3}$ uygulamalarında daha belirgin olduğu görülmektedir (Çizelge 1 ve 2). Tohum uygulamalarının birçok türde çimlenmeyi hızlandırdığına dair çalışma (Harris et al
2001; Basra et al 2005; Farooq et al 2007; 2009; Kaya et al 2010) sonuçları nettir. Tohum uygulamaları ile kökçük çıkışına (3. aşama) kadar olan çimlenme aşamasının (1. ve 2. aşama) tamamlanmış olması hızlı çimlenmede etkili faktör olmuştur.

Fide sap boyunun her iki bitkide $\mathrm{GA}_{3}$ uygulamaları ile kontrole göre artış göstermesi önceki çalışma (Atar 2010) sonuçları ile uyum göstermektedir. Hidroprimig uygulamasında ise fide sap boyu buğdayda Basra et al (2005) belirttiklerinin aksine kontrole göre azalırken, arpada artmıştır. Basra et al (2005) hidropriming uygulamasının kök uzunluğunu arttırdığını belirtmişlerdir. Çalışmamızda ise fide kök boyu buğdayda tohum uygulamaları ile değişmezken, arpada en yüksek hidropriming uygulamasında belirlenmiştir. Buğdayda bu sonucun görülmemesinin 'kök büyümesi üzerine $\mathrm{GA}_{3}$ etkisi önemli olsa da diğer hormonlarla kıyaslandığında belirgin değildir' sonucuyla (Tanimoto 2005) uyum göstermektedir. Ayrıca Leite et al (2003) $\mathrm{GA}_{3}$ uygulamasının soyada ilk dönem kök büyümesini geciktirdiğini belirtmişlerdir.

Fide yaş ve kuru ağırlığı bakımından buğdayda $\mathrm{GA}_{3}$ uygulamaları olumlu etkide bulunmuştur. $\mathrm{Bu}$ duruma $\mathrm{GA}_{3}$ uygulamalarının çıkış süresi üzerindeki olumlu etkisinin yansıması olduğu düşünülmektedir. Hidropriming uygulaması ise Basra et al (2005) ve Rafiq et al (2006) belirttiğinin aksine olumlu etki göstermemiştir. GA $\mathrm{G}_{3}$ uygulamasının fide kuru ağırlığı üzerine olumlu etkisi (Tanimoto 1990; Datta et al 1997/98) önceki çalışmalarda da belirlenmiştir. Arpada uygulamaların fide yaş ve kuru ağırlığı üzerine etkisi olmamıştır.

\section{Sonuçlar}

Araştırmada buğdayın yüksek ve düşük toprak nem düzeyine hassas olmasına rağmen, arpanın daha toleranslı olduğu görülmüsştür. Her iki bitkide en yüksek çimlenme oranı yüzdeleri $\mathrm{S}_{3}$ nem düzeyinde bulunurken, arpada hızlı çıkış ve fide gelişimi için en iyi toprak tavının tarla kapasitesi $\left(\mathrm{S}_{1}\right)$, buğday için ise $\mathrm{S}_{2}$ nem düzeyi olduğu söylenebilir. Ekim öncesi tohum uygulamalarının çalışmamızda olduğu gibi, olumlu etkisine dair fazla sayıda araştırma bulunmaktadır. Olumsuz etkisi belirtilmemiş ve uygulaması fazla maliyet gerektirmeyen, özellikle düşük dozlu (30 ppm) $\mathrm{GA}_{3}$ içerikli tohum uygulamalarının kullanılmasının yaygınlaştırılması faydalı olacaktır. 


\section{Kaynaklar}

Afzal I, Basra S M A \& Iqbal A (2005). The effects of seed soaking with plant growth regulators on seedling vigor of wheat under salinity stress. Journal of Stres Physiology \& Biochemistry 1(1): 6-14

AOSA 1983. Association of Official Seed Analysis (AOSA), Seed Vigor Testing Handbook. Contribution No: 32 to the handbook on Seed Testing

Atar B (2010). Bazı ekmeklik buğday (Tritucum aestivum L) çeşitlerinde tohuma ön işlem ve azot dozu uygulamalarının kış öncesi büyüme özellikleri ile tane verimi ve kalite özelliklerine etkileri. Doktora tezi, Süleyman Demirel Üniversitesi Fen Bilimleri Enstitüsü (Basılmamış), Isparta

Basra S M A, Afzal I, Rashid R A \& Farooq M (2005). Pre-sowing seed treatments to improve germination and seedling growth in wheat (Triticum aestivum L.). Caderno de Pesquisa Serie Biologia 17(1): 155-164

Coolbear P, Francis A \& Grierson D (1984). The effect of low temperature pre-sowing treatment under the germination performance and membrane integrity of artificially aged tomato seeds. Journal of Experimental Botany 35: 1609-1617

Datta K S, Varma S K, Angrish R, Kumar B \& Kumar1 P (1997/98). Alleviation of salt stres by plant growth regulators in Triticum aestivum L. Biologia Plantarum 40: 269-275

Ellis R H \& Roberts E H (1981). The quantification of ageing and survival in orthodox seeds. Seed Science and Technology 9: 373-409

Farooq M (2005). Assessment of physiological and biochemical aspects of pre-sowing seed treatments in transplanted and direct seeded rice. Faculty of Agriculture, Phd. Thesis, pp. 286, Faisalabad, Pakistan

Farooq M, Basra S M A, Rehman H \& Saleem B A (2007). Seed priming enhances the performance of late sown wheat (Triticum aestivum L. ) by improving chilling tolerance. Journal of Agronomy and Crop Science 194 (1): 55-60

Farooq M, Basra S M A, Wahid A, Khaliq A \& Kobayashi N (2009). Rice seed invigoration. In: E. Lichtfouse (ed.) Sustainable Agriulture Reviews. pp. 137-175, Springer. The Netherlands

Giri G S \& Schillinger W F (2003). Seed priming winter wheat for germination, emergence, and yield. Crop Science Society of America 43: 2135-2141

Harris D, Breese W A \& Kumar Rao J V D K (2005). The improvement of crop yield in marginal environments using 'on-farm' seed priming: nodulation, nitrogen fixation, and disease resistance. African Journal of Agricultural Research 56: 1211-1218
Harris D, Raghuwanshi B S, Gangwar J S, Sing S C, Joshi K D, Rashid A \& Hollington P A (2001). Participatory evaluation by farmers of on-farm seed priming in wheat in India, Nepal and Pakistan. Experimental Agriculture 37: 403-415

Heydecker W \& Coolbear P (1977). Seed treatment for improved performance. survey and attempted prornosis. Seed Science and Technology 5: 353-425

İdris M \& Aslam M (1975). The effect of soaking and drying seeds before planting on the germination and growth of Triticum vulgare under normal and saline conditions. Canadian Journal of Botany 53(13): 1328-1332

Kaya G, Demir İ, Tekin A, Yaşar F \& Demir K (2010). Priming uygulamasının biber tohumlarının stres sicaklıklarında çimlenme, yağ asitleri, şeker kapsamı ve enzim aktivitesi üzerine etkisi. Tarım Bilimleri Dergisi- Journal of Agricultural Sciences 16(1): 9-16

Khan A A (1992). Preplant physiological seed conditioning. Horticulture Review 13: 131-181

Leite V M, Rosole C A \& Rodrigues J D (2003). Gibberellin and cytokinin effects on sobean growth. Scienta Agricola 60(3): 537-541

Lindstrom M J, Papendick R I \& Koehler F E (1976). A Model to predict winter wheat emergence as affected by soil temperature, water potential, and deph of planting. Agronomy Journal 68: 137-140

Özbingöl N, Corbineau F, Groot S P C, Bino R J \& Come D (1999). Activation of the cell cycle in tomato (Lycopersicon esculentum Mill.) seeds during osmoconditioning as related to temperature and oxygen. Annals of Botany 84: 245-251

Rafiq S, Iqbal T, Hameed A, Rafiq1 Z A \& Rafiq N (2006). Morphobiochemical analysis of salinity stress response of wheat. Pakistan Journal of Botany 38(5): 1759-1767

Shahzad M A, Din W U, Sahi S T, Sahi S T, Khan M M \& Ahmad E M (2007). Effect of sowing dates and seed treatment on grain yield and quality of wheat, Pakistan Journal of Agricultural Sciences 44(4): 581583

Tanimoto E (1990). Gibberellin requirement for the normal growth of roots. In $\mathrm{N}$ Takahashi, B Phinney, J Mac-Millan, eds, Gibberellins. Springer-Verlag, New York, pp. 229-240

Tanimoto E (2005). Regulation of root growth by plant hormones-roles for auxin and gibberellin. Critical Reviews in Plant Sciences 24(4): 249-265

Wurr D \& Fellows J (1983). The effect of the time of seedling emergence of crisp lettuce on the time of maturity and head weight at maturity. Journal Horticultural Science 58: 561-566 